\title{
Antitumor effect of dendritic cells transfected with prostate-specific membrane antigen recombinant adenovirus on prostate cancer: An in vitro study
}

\author{
FAN-DONG MENG, SHUAI WANG, YOU-HONG JIANG and CHENG-GUANG SUI
}

Cancer Research Institute, The First Affiliated Hospital, China Medical University, Shenyang, Liaoning 110001, P.R. China

Received November 25, 2014; Accepted September 25, 2015

DOI: $10.3892 / \mathrm{mmr} .2016 .4754$

\begin{abstract}
The aim of the present study was to construct a dendritic cell (DC) vaccine transfected with a prostate-specific membrane antigen (PSMA) recombinant adenovirus and to observe the ability of the recombinant DCs in eliciting a PSMA-directed T-cell response to prostate cancer (PCa) in vitro. Replication-defective adenoviral vectors, were constructed using the Adxsi system. The virus titer was measured by TCID50 assay, and the expression of the PSMA gene was identified by polymerase chain reaction (PCR) generation of peripheral blood mononuclear cell (PBMC) derived DCs in vitro. In addition, a PE-7AAD apoptosis and necrosis kit was applied to detect the survival of DCs at different MOI values to determine the optimal MOI. Morphological changes of DC were observed under a fluorescence microscope, expression of the PSMA protein was detected by western blotting $48 \mathrm{~h}$ after transfection, the expression of DC markers prior to and following transfection was detected by direct immunofluorescence, and the interleukin (IL)-12 concentration in the culture supernatant of the three groups was measured by ELISA. The antitumor effect of DC-stimulated cytotoxic T lymphocytes (CTLs) on different PCa cell lines was analyzed using a Cell Counting Kit- 8 assay. The optimal MOI value was determined to be 200. The PSMA protein was expressed in DCs, and the recombinant adenovirus did not impact the maturation and morphological changes of the DCs. The expression levels of the co-stimulatory molecules, CD80, CD83, CD86 and HLA-DR, were significantly higher in the Ad-PSMA-DC group than in the other two groups $(\mathrm{P}<0.05)$. The concentration of IL-12 in the supernatant of the Ad-PSMA-DC group $(79.51 \pm 1.60 \mathrm{pg})$ was comparable with that of the Ad-GFP-DC group (not significantly different), and the two were significantly higher
\end{abstract}

Correspondence to: Dr Cheng-Guang Sui, Cancer Research Institute, The First Affiliated Hospital, China Medical University, 155 Nanjing North Street, Heping, Shenyang, Liaoning 110001, P.R. China

E-mail: suichengguang_1@126.com

Key words: dendritic cell, prostate-specific membrane antigen, cytotoxic T lymphocyte, prostate cancer than the non-transfection group $(\mathrm{P}<0.05)$. The optimal effector to target (E:T) ratio was determined to be 40:1. However, at the same E:T ratio, the cytotoxic activity of PSMA-DC-T against the LNCap cells was markedly stronger than its activity against the other target cells (DU145 and 22RV; $\mathrm{P}<0.05$ ); furthermore, the the cytotoxic activity of PSMA-DC-T against the LNCap cells was significantly higher than the anti-LNCap effect of DC-T cells in other groups $(\mathrm{P}<0.05)$. In vitro experiments indicated that mature DCs transfected with Ad-PSMA secreted high concentrations of IL-12 and elicited potent antitumor immune responses targeting PSMA-expressing cells by stimulating the cytotoxic activity of CTLs.

\section{Introduction}

In the history of tumor immunotherapy, dendritic cell (DC) vaccines targeting cancer cells have been a significant focus of investigation (1). Abundant animal experiments, and phase I and II clinical trials have demonstrated that DCs elicit specific antitumor immune responses, which lead to tumor regression or stabilization (2). DC vaccines have entered phase I or II in clinical trials regarding the treatment of various types of tumor (1).

Prostate cancer ( $\mathrm{PCa}$ ) is the second most common type of malignancy in men in western countries (3) and the incidence of $\mathrm{PCa}$ in China has recently risen (4). Treatment of $\mathrm{PCa}$ predominantly comprises of surgery, radiotherapy, chemotherapy and endocrine therapy, which exert certain therapeutic effects, yet are accompanied by serious adverse reactions, particularly to patients exhibiting sex hormone-independent $\mathrm{PCa}$, and recurrence and/or metastasis.

$\mathrm{DC}$ vaccine therapy has become a significant focus for $\mathrm{PCa}$ immunotherapy and also in the field of biotherapy as a whole. Studies in China and worldwide have evaluated DC vaccines [loaded with prostate-associated antigen peptides, including prostate-specific antigen (PSA), prostate-specific membrane antigen (PSMA), prostatic acid phosphatase (PAP), prostate stem cell antigen, related recombinant protein and tumor cell lysates] in pre-clinical studies, and confirmed their safety and therapeutic effect of stimulating specific immune responses against PCa (5-7). Despite the progression of the DC vaccine in basic and clinical research, various issues remain to be clarified, such as the instability of the therapeutic effect, the selection of an appropriate tumor antigen, the route and dose 
of reinfused DCs, the selection of a gene transfection vector, and the extension of the response time of the vaccine-induced cytotoxic T lymphocyte (CTL). The present study hypothesizes that the DC vaccine presents a novel therapeutic method for the treatment of $\mathrm{PCa}$, particularly for individuals with hormone resistance.

Currently, there are two strategies for constructing DC vaccines (8): i) DCs loaded with tumor antigen; and ii) DCs modified with cytokines. The former aims to elevate the targeting effect of immunotherapy, and predominantly consists of DCs pulsed with tumor antigens and DCs pulsed with antigen coding genes. However, the latter focuses on improving the efficacy of DC vaccines by introducing co-stimulatory molecules, cytokines and chemokines into the DCs. Of these methods, DCs modified by antigen coding genes effectively present tumor antigens to $\mathrm{T}$ cells subsequent to stimulation and continue to release endogenous tumor antigens. Therefore, DCs modified by antigen coding genes are considered to be more effective in stimulating $\mathrm{T}$ lymphocytes (in terms of a longer duration of antigen presenting and an improved targeting effect) when compared with DCs pulsed directly by tumor antigens or tumor cell debris. In addition, numerous studies have identified the association between DC function and a variety of cytokines, co-stimulatory molecules, and chemokines (9-11). Therefore, introducing various cytokines into DCs in order that they continue to express these factors has become an additional method of promoting DC maturation, improving DC activity, enhancing the antigen-presenting function (by increasing the expression level of co-stimulatory molecules), and strengthening the chemotaxis of DCs to T cells.

PSMA is a type of PCa marker, which is more sensitive and more specific when compared with PSA and PAP. Its highly specific expression in PCa tissues has rendered it a primary target for targeted therapy and a specific focus within PCa research (12). DCs are considered to be the most potent antigen-presenting cells in current knowledge. DCs stimulate and sensitize naive $\mathrm{T}$ cells in the initiation of an early immune response. Furthermore, DCs elicit immunization targeting PCa cells upon ingestion and processing of tumor antigens, and presentation to T cells (13).

The selection of a vector is another key issue in the construction of DC vaccines targeted to PCa cells. Currently, gene therapy vectors consist of two major categories, viral vectors (adenoviruses, retroviruses, adeno-associated viruses, lentiviruses and the herpes simplex virus) and non-viral vectors (bacterial vectors, artificial carriers and liposome vectors). Non-viral vectors have no limitations in the size of inserted elements, possess low immunogenicity and are easy to produce; however, their low transfection efficiency in vivo has reduced their popularity compared with viral vectors (14). Among the widely used viral vectors, the adenovirus system is considered to be an effective vector for introducing exogenous genes and small interfering RNA into all types of cells (dividing and non-dividing) (15). Adenoviruses possess a high transfection efficiency ( 100\%), low genotoxicity, a marginal possibility of carcinogenic mutation (its genome is not integrated into the host cell genome), and is easy to use with recombinant DNA techniques. In addition, the adenoviral genome is large (36 kb), therefore, the insertion of larger fragments is straightforward.
Furthermore, by using adenoviral vectors, a transfection efficiency of 50 90\% is more likely without inhibiting DC activity and immune function. However, the adenovirus system is not flawless; the reduced duration of action in transient transfection and the immunogenicity of the adenovirus vector remain to be resolved $(16,17)$.

Thus, the present study aims to construct a pAdxsi-GFP-PSMA adenovirus and develop a novel type of $\mathrm{DC}$ vaccine to target $\mathrm{PCa}$, as well as to investigate its therapeutic effect in vitro. This may enable its further application in vivo and provide a novel approach for DC-based immunotherapy for the treatment of PCa.

\section{Materials and methods}

Reagents. Restriction endonuclease was purchased from New England Biolabs (UK) Ltd., Co., (Hitchin, UK); T4 DNA ligase was purchased from JingMei Biological Engineering Co., Ltd. (Shenzhen, China); Alkaline Phosphatase, Calf Intestinal (CIP) enzyme was obtained from Promega Corporation (Madison, WI, USA); Invitrogen Pfx DNA polymerase was purchased from Thermo Fisher Scientific, Inc. (Waltham, MA, USA); dNTP was purchased from Sangon Biotec Co., Ltd. (Shangai, China) and primers were synthesized by Sangon Biotec Co., Ltd. A DNA purification kit (column-type centrifugal), and a DNA gel extraction and purification kit (column-type centrifugal) were purchased from Zweig Las Biotechnology Co., Ltd. (Beijing, China); a 1-kb DNA ladder was purchased from DingGuo Biotech., Co. (Shanghai, China); DNA Marker DL2000 was obtained from Takara Biotechnology Co., Ltd. (Dalian, China); the total protein cleaving enzyme and protease inhibitors were purchased from Beyotime Institute of Biotechnology (Haimen, China); a three-color pre-stained protein marker was purchased from Beyotian Biotechnology Co. (Shanghai, China); rabbit anti-human PSMA monoclonal antibody and rabbit anti-human $\beta$-actin polyclonal antibody were purchased from Jiangsu Kangwei Biological Co., Ltd. (Dongtai, China). RPMI-1640 and fetal bovine serum (FBS) were purchased from Thermo Fisher Scientific Inc. (Gibco); recombinant human granulocyte-macrophage colony-stimulating factor (GM-CSF), recombinant human interleukin (IL)-4, IL-2 and tumor necrosis factor (TNF) were purchased from Tebao Biological engineering Co., Ltd. (Xiamen, China). Lymphocyte separation medium was purchased from Haoyang Biotechnology Co., Ltd. (Tianjin, China); phosphate-buffered saline (PBS) was purchased from Boster Biological Technology, Ltd. (Wuhan, China); phycoerythrin (PE)-labeled mouse anti-human CD80 (cat no. 557227) and CD86 (cat. no. 560957)antibodies, peridinium chlorophyll protein (PerCP)-labeled mouse anti-human HLA-DR antibody (Cat. no. 551375) and allo-phycocyanin (APC)-labeled mouse anti-human CD83 antibody (cat. no. 551073) were purchased from BD Pharmingen (San Diego, CA, USA). The Cell Counting Kit-8 (CCK-8) assay kit was purchased from Skylight Biotech Co., Ltd. (Akita, Japan); interferon (IFN)- $\gamma$ and IL-10 ELISA kits were purchased from Dakewe Biotech Co., Ltd. (Shenzhen, China) and a PE/7AAD apoptosis kit was purchased from KeyGen Biotech. Co., Ltd. (Nanjing, China). 
Target gene, vector plasmid and cell strains. A cDNA plasmid for pCMV-SPORT6/PSMA construction was purchased from Mitaka Biotechnology Co., Ltd (Wuhan, China). The shuttle plasmid for pShuttle-CMV-EGFP (Shanghai Jikai Gene Chemical Technology Co. Ltd., Shanghai, China), pAdxsi adenovirus backbone plasmid (Shanghai Jikai Gene Chemical Technology Co. Ltd.), super chemically competent Escherichia coli DH5a and HEK293 cells (Shanghai Jikai Gene Chemical Technology Co. Ltd.) were maintained in the laboratory at China Medical University (Shenyang, China). The PCa cell strains, LNCap, Du145 and 22RV were also preserved in this lab.

Construction and identification of PSMA adenovirus plasmid. Using PSMA as the reference sequence, two primers, containing SfiI restriction sites at the 5'-end, were designed as follows: 5'-AAAAGGCCGCTGCGGCCACCATGTGGAA TCTCCTTCA-3' for P1 and 5'-AAAAGGCCTGTTTGGC CTTAGGCTACTTCACTCA-3' for P2.

Construction and identification of shuttle plasmid, pShuttle-GFP-PSMA. pCMV-SPORT6/PSMA served as a template, and $\mathrm{P} 1, \mathrm{P} 2$, and the $P f u$ enzyme (pfx DNA polymerase) were used for polymerase chain reaction (PCR) amplification. The amplified product was processed by SfiI digestion to obtain 2.2-Kb PSMA gene fragments. The adenovirus shuttle plasmid, pShuttle-CMV-EGFP was also digested with SfiI, and agarose gel electrophoresis was performed to retrieve a 5.1-Kb vector DNA. The amplified PSMA gene and retrieved vector DNA fragment were joined using T4 DNA ligase at $22^{\circ} \mathrm{C}$ for $4 \mathrm{~h}$ (molecular concentration ratio, 3:1). The products were transformed into chemically competent $E$. coli DH5 $\alpha$. The positive clones were selected using kanamycin (Shenyang Boermei Reagent Co., Shenyang, China), and underwent DNA sequencing following identification by restriction enzyme digestion. The recombinant shuttle plasmid, pShuttle-GFP-PSMA and the empty vector, pShuttle-CMV-EGFP were digested by SfiI, and underwent $1 \%$ agarose gel electrophoresis to identify any PSMA gene fragments. Additionally, the recombinant vector, pShuttle-GFP-PSMA was sent to Shanghai Shenggong Co., Ltd. (Shangai, China) for sequencing to ensure the insertion sequence and reading frame were correct.

Construction and identification of recombinant adenovirus plasmid, pAdxsi-GFP-PSMA. The shuttle plasmid, pShuttle-GFP-PSMA and the pAdxsi vector were processed by I-CeuI and I-SceI double digestion. The target gene of the shuttle plasmid was retrieved on gel, while the digested vector was collected using an ethanol precipitation method following CIP dephosphorylation. The treated vector fragment and the insertion element were joined by T4 DNA ligase, and the 3- $\mu 1$ ligation product was transformed into a chemically competent DH5 $\alpha$ cell strain and smeared onto an ampicillin-resistant solid-medium plate. Positive clones were seeded onto the ampicillin-resistant liquid medium and incubated overnight at $4^{\circ} \mathrm{C}$. The plasmid was purified using a small/medium quantity plasmid extraction purification kit, and the recombinant adenovirus plasmid, pAdxsi-GFP-PSMA was obtained. XhoI enzyme digestion was performed, followed by $1 \%$ agarose gel electrophoresis, to identify whether the recombinant adenovirus plasmid had been constructed correctly.

Packaging, amplification and purification of recombinant human PSMA adenovirus. HEK293 cells were cultured in Dulbecco's modified Eagle's medium (Shenyang Boermei Reagent Co.) containing $10 \% \mathrm{FBS}$ and incubated at $37^{\circ} \mathrm{C}$ in an atmosphere of $5 \% \mathrm{CO}_{2}$. The cells were transfected with the recombinant adenovirus at a density of $80-90 \%$. For transfection, an adenovirus vector plasmid carrying the PSMA gene was linearized with the restriction enzyme $P a c I$ and Lipofectamine 2000 (Shenyang Boermei Reagent Co.) was used to mediate the transfection and packaging of the HEK293 cells. The packaged cells were incubated at $37^{\circ} \mathrm{C}$ in an atmosphere of $5 \% \mathrm{CO}_{2}$ for 7-12 days before cytopathic effects (CPEs) and green fluorescent protein (GFP) could be observed under an ordinary microscope and a fluorescence microscope, respectively. The cells were collected, centrifuged at $800 \mathrm{x} \mathrm{g}$ for $5 \mathrm{~min}$, resuspended with $1 \mathrm{ml} \mathrm{PBS}$, then underwent three cycles of repeated freezing and thawing $\left(-80^{\circ} \mathrm{C}\right.$ to $\left.37^{\circ} \mathrm{C}\right)$. The supernatant containing the recombinant viruses was collected following centrifugation. The above-mentioned supernatant (40-50\%) was used for re-transfection of the HEK293 cells, and the same freeze/thaw method was repeated four times before the supernatant was collected and the virus was purified by $\mathrm{CsCl}$ density gradient centrifugation. The virus was divided into small portions and preserved at $-80^{\circ} \mathrm{C}$ for subsequent experiments.

Extraction, identification and titer determination of recombinant adenovirus plasmid. The recombinant virus supernatant $(10 \mu \mathrm{l})$ was added to proteinase $\mathrm{K}(10 \mathrm{mg} / \mathrm{ml})$, incubated at $50^{\circ} \mathrm{C}$ for $1 \mathrm{~h}$ and boiled at $100^{\circ} \mathrm{C}$ for $5 \mathrm{~min}$. Following centrifugation, $2 \mu \mathrm{l}$ supernatant served as a template for PCR identification. The HEK293 cells were plated in 96-well plates and the concentrated virus solution was added into the cultured cells at different dilutions. The infected cells were incubated at $37^{\circ} \mathrm{C}$ for 10 days and the number of wells per line that were exhibiting CPE was recorded under a fluorescence microscope. The ratio of positive wells was calculated. According to the 50\% Tissue Culture Infectious Dose (TCID50) formula method, for a $100-\mu 1$ sample, the titer $(\mathrm{T})=10^{1+\mathrm{d}(\mathrm{s}-0.5)}$ (where $\mathrm{d}$ is $\log 10$ dilution and $\mathrm{s}$ is the sum of the ratio of positive wells at each dilution). In order to convert from TCID50/ml to $\mathrm{pfu} / \mathrm{ml}$ the following formula was used: $\mathrm{T}=\mathrm{a} \times 10^{\mathrm{b}} \mathrm{TCID} 50 / \mathrm{ml}=\mathrm{a} \times 10^{\mathrm{b}-0.7} \mathrm{pfu} / \mathrm{ml}$.

Induction of peripheral blood mononuclear cell (PBMC)-derived dendritic cells and determination of the efficiency of adenovirus infection in vitro. Blood cell separator was applied for separation and enrichment of PBMCs obtained from ten healthy volunteers (mean age, $39 \pm 5$ years; 3 male, 7 female). Blood collection performed at The First Affiliated Hospital, China Medical University (Shenyang, China). Ficoll lymphocyte separation medium was used with density gradient centrifugation for preliminary purification of the mononuclear cells. The cells were rinsed twice with PBS, counted and stained with trypan blue (Shenyang Boermei Reagent Co.) to detect cell viability. The cells were resuspended with RPMI-1640 medium containing 10\% FBS at 
a density of $2 \times 10^{6} / \mathrm{ml}$ and seeded in $25-\mathrm{cm}^{2}$ flasks. The cells were incubated at $37^{\circ} \mathrm{C}$ in an atmosphere of $5 \% \mathrm{CO}_{2}$ for $2 \mathrm{~h}$, and the non-adherent cells were extracted into another culture flask for CTL induction in vitro. The adherent cells were cultured in RPMI-1640 medium containing 10\% FCS, with $100 \mathrm{ng} / \mathrm{ml} \mathrm{GM-CSF}$ and $50 \mathrm{ng} / \mathrm{ml} \mathrm{IL-4}$. Half of the medium was changed every three days and fresh cytokines were added. After six days of induction, 1,000 U/ml TNF- $\alpha$ was added to stimulate DC maturation and the mature DCs were collected on day seven or eight.

Transfection of the empty viral plasmid, Ad-GFP into immature DCs and detection of transfection efficiency at different MOI. On day five, immature DCs that were half-adherent were collected and seeded in 24-well plates in $0.2 \mathrm{ml}$ medium containing $10 \%$ FBS $\left(5 \times 10^{5}\right.$ cells/well). The concentration of Ad-GFP was adjusted to $2 \times 10^{7} \mathrm{pfu} / \mu \mathrm{l}$. The immature DCs were divided into five groups (three wells per group), and the Ad-GFP virus was added to the DCs in each group at MOI values of 50,100,200, 300 and 400. DCs containing the virus were incubated at $37^{\circ} \mathrm{C}$ in $5 \% \mathrm{CO}_{2}$ for $2 \mathrm{~h}$, during which the culture plates were agitated every $15 \mathrm{~min}$ to ensure uniform infection of the virus. Then RPMI-1640 medium containing $10 \%$ FCS, $100 \mathrm{ng} / \mathrm{ml} \mathrm{GM-CSF}$ and $50 \mathrm{ng} / \mathrm{ml} \mathrm{IL-4} \mathrm{was} \mathrm{added}$ to each well and the cells were observed under a fluorescence microscope at 12, 24 and $48 \mathrm{~h}$ to detect the expression of GFP at one MOI value. At $48 \mathrm{~h}$, flow cytometry was used to detect the GFP-positive rate at different MOI values, and a PE-7AAD apoptosis and necrosis kit was used to detect the apoptosis rate of the DCs, in order to determine the optimal MOI.

Changes in DC immune function following infection with the PSMA gene recombinant adenovirus

$D C$ and CTL culture in vitro. The method of DC culture is described above. The non-adherent cells were seeded in an additional flask and differentiated into CTLs in the presence of IL-2.

Expression of PSMA in recombinant adenovirus-mediated $D C s$. On the fifth day, half-adherent immature DCs were collected and made into a single cell suspension. The appropriate quantities of Ad-PSMA and Ad-GFP were added into the above-mentioned DC suspension according to the optimal MOI value, creating two groups of DCs: The Ad-PSMA-DC and Ad-GFP-DC groups. After $48 \mathrm{~h}$ of incubation at $37^{\circ} \mathrm{C}$ in a $5 \% \mathrm{CO}_{2}$ atmosphere cells were processed with radioimmunoprecipitation assay lysis buffer and protease inhibitors. The cell lysate was centrifuged at $4^{\circ} \mathrm{C}$ for $5 \mathrm{~min}$ at $800 \mathrm{x}$ g, and the supernatant was transferred to a clean Eppendorf (EP) tube and preserved at $-20^{\circ} \mathrm{C}$. Separating gel $(10 \%)$ and $5 \%$ stacking gel were prepared for electrophoresis. The protein was transferred onto a polyvinylidene fluoride membrane (Shenyang Boermei Reagent Co.) and the membrane was blocked with $5 \%$ skimmed milk at room temperature for $2 \mathrm{~h}$ on a thermostatic shaker. The primary antibody (rabbit anti-human PSMA monoclonal antibody) was added at a concentration of 1:2,000 in Tris-buffered saline with Tween-20 (TBST) and incubated at $4^{\circ} \mathrm{C}$ overnight. The membrane was washed with TBST, three times for 10 min each time, and the horseradish peroxidase (HRP)-labeled secondary antibody (HRP-goat anti-rabbit
$\mathrm{IgG}$ ) was added and the membrane was incubated at room temperature for $1 \mathrm{~h}$. The membrane was washed with TBST a further three times (10 min each time), and visualized in a dark room by enhanced chemiluminescence.

Detection of DC phenotype before and after virus infection by direct immunofluorescence. Fluorescent antibodies were PE-labeled CD80 and CD86, peridinin chlorophyll-labeled HLA-DR and allophycocyanin-labeled CD83. Immature DCs on day 5, mature DCs on day 8 and the cells infected with adenovirus for $48 \mathrm{~h}$ (Ad-PSMA-DC and Ad-GFP-DC) were collected. The four types of cell were rinsed twice with PBS, centrifuged at $800 \mathrm{x}$ g for $5 \mathrm{~min}$ and the cell density was adjusted to $\sim 1 \times 10^{6} / \mathrm{ml}$. Each type of cell suspension $(1 \mathrm{ml})$ was transferred to four EP tubes and the fluorescent antibodies were added. After $30 \mathrm{~min}$ of incubation at $4^{\circ} \mathrm{C}$, cells in each tube were rinsed twice with PBS, resuspended with $0.5 \mathrm{ml}$ PBS and transferred into FACS tubes (BD Pharmingen) for phenotype analysis.

Detection of the IL-12 level in the supernatant of the DC vaccine in each group by ELISA. On the fifth day of DC culture, the recombinant virus was added to each group according to the optimal MOI value and the DCs were divided into three groups: Ad-PSMA-DC, Ad-GFP-DC and normal DCs. On the sixth day, 1,000 U/ml TNF- $\alpha$ was added to each group for DC maturation stimulation. The supernatant of each group was collected after $48 \mathrm{~h}$ of transfection, and the quantity of IL-12 in each group was detected according to the ELISA kit instructions.

Detection of CTL cytotoxicity to the three PCa cell lines (LNCap, Dul45 and 22RV) in the different groups by CCK-8 assay. On the fifth day of DC culture, a recombinant virus was added to each group according to the optimal MOI value and the DCs were divided into three groups: Ad-PSMA-DC, Ad-GFP-DC and normal DCs. The DCs were incubated at $37^{\circ} \mathrm{C}$ with $5 \%$ $\mathrm{CO}_{2}$ for $48 \mathrm{~h}$ to form stimulation cells. Prior to use, $10 \mu \mathrm{g} / \mathrm{ml}$ mitomycin was added and the cells were incubated at $37^{\circ} \mathrm{C}$ for 30 min, rinsed with RPMI-1640 incomplete medium (serum free) three times and adjusted to a density of $1 \times 10^{6} / \mathrm{ml}$. The non-adherent mononuclear cells were cultured in RPMI-1640 medium containing $10 \% \mathrm{FBS}$ and $1,000 \mathrm{U} / \mathrm{ml} \mathrm{IL}-2$. Every three days, fresh medium and IL-2 were added to induce CTLs, the cell density was adjusted to $1 \times 10^{6} / \mathrm{ml}$ and prepared as reaction cells. The above-mentioned stimulation cells (each group of DCs) and reaction cells (CTLs) were co-cultured at a ratio of 1:10 in complete medium at a density of $2 \times 10^{6} / \mathrm{ml}$ for $96 \mathrm{~h}\left(37^{\circ} \mathrm{C}, 5 \%\right.$ $\mathrm{CO}_{2}$ ). In addition, the PCa cells (LNCap, Du145 and 22RV) at the logarithmic growth phase were plated in 96-well plates and cultured for $24 \mathrm{~h}$ for cell attachment. The DC-CTL of the three groups and the CTL control were added to the plates containing cancer cells at different effector to target (E:T) ratios (10:1, 20:1 or 40:1) and the mixtures were incubated for another $24 \mathrm{~h}$ before the CCK-8 reagent was added (concentration, $20 \mu \mathrm{l} /$ well). After $4 \mathrm{~h}$ of incubation at $37^{\circ} \mathrm{C}$, the optical density (OD) value of each well was detected with ELISA and the cytotoxicity of CTLs in each group was calculated according to the following formula: Killing rate $(\%)=\left[1-\left(\mathrm{OD}_{\text {experimental group }}-\mathrm{OD}_{\text {effector cells alone }}\right) / \mathrm{OD}_{\text {target }}\right.$ cell alone] x 100. 


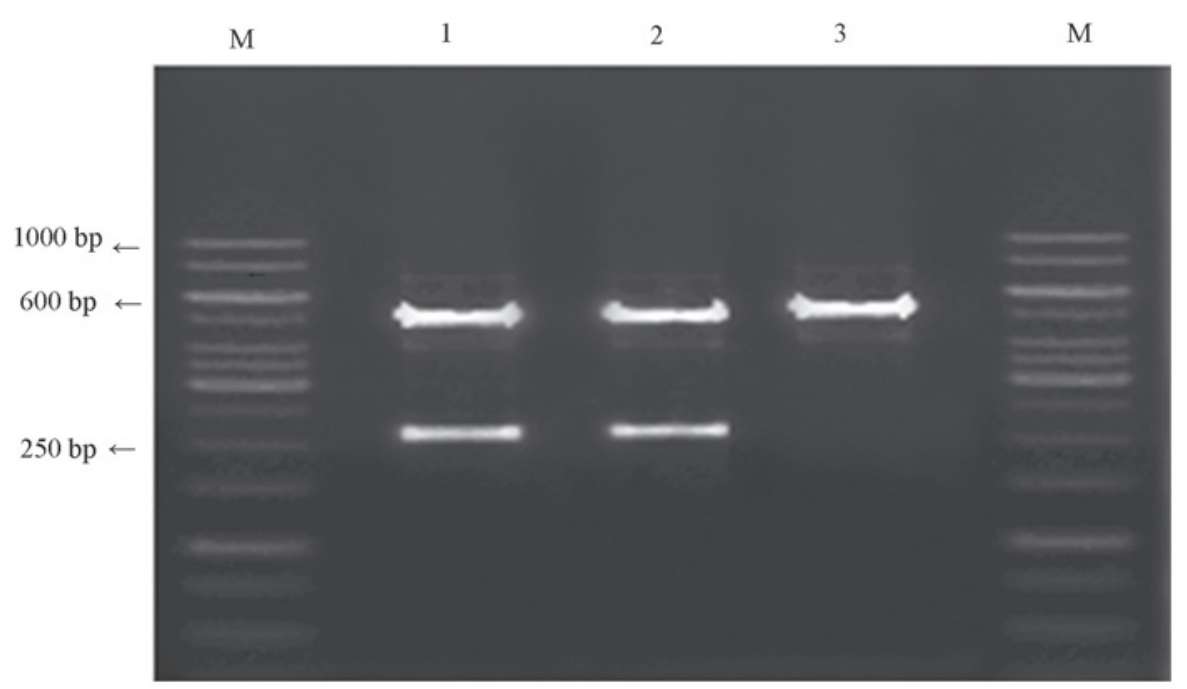

Figure 1. Identification results of pShuttle-GFP-FOLH1. M, marker; 1, shuttle plasmid; 2, pShuttle-GFP-PSMA; 3, empty vector.

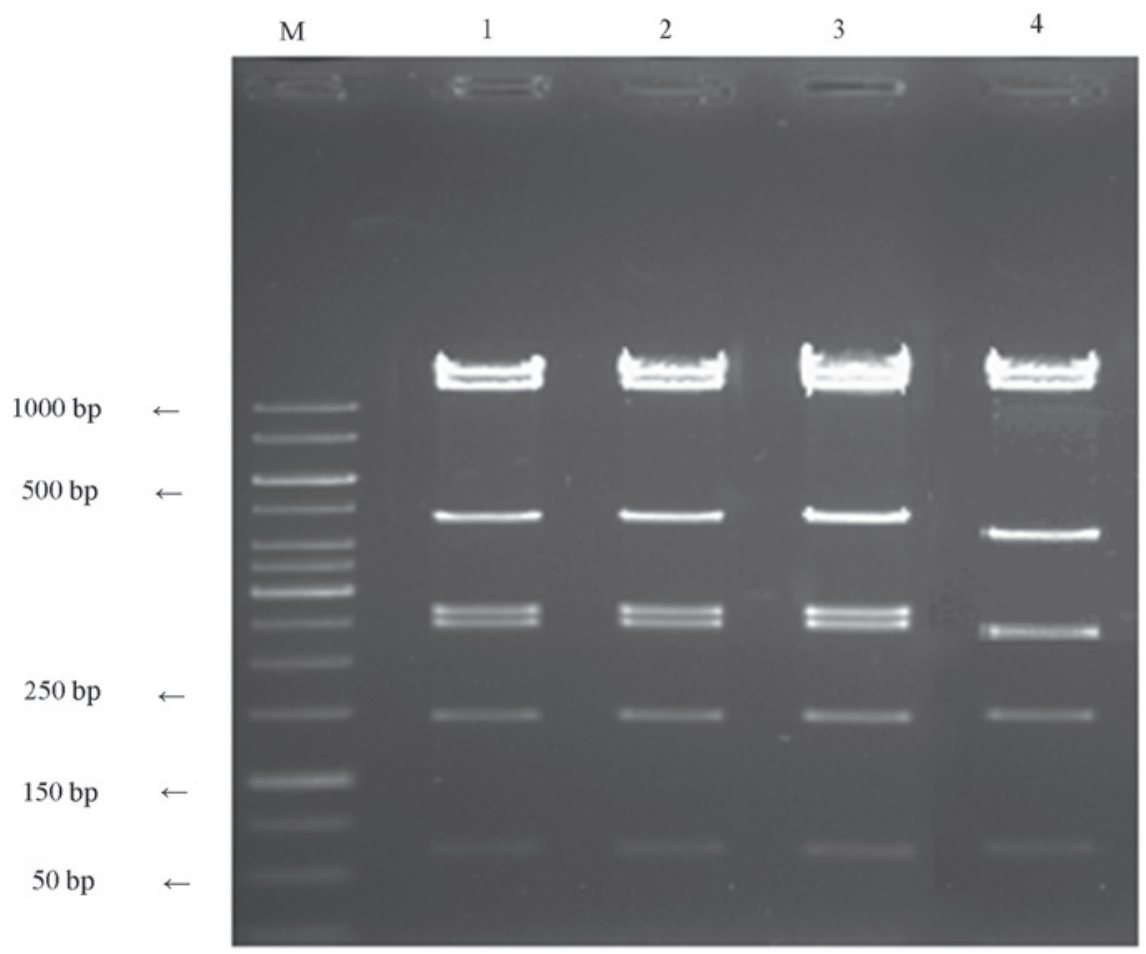

Figure 2. Identification results of pAdxsi-GFP-FOLH1. Lane M, marker (1 kb DNA ladder); lanes 1-3, positive clones 14, 11.8, 4.8, 2.66, 2.47 , 1.45 and 0.6 к; lane 4, negative clones 14, 11.8, 4.0, 2.47, 1.45 and $0.6 \mathrm{~kb}$.

Statistical analysis. Data were processed with SPSS 16.0 software (SPSS, Inc., Chicago, IL, USA) and expressed as means \pm standard deviation. Multiple comparisons of sample means were analyzed by one-way analysis of variance and $\mathrm{P}<0.05$ was considered to indicate a statistically significant difference.

\section{Results}

Identification of shuttle plasmid, pShuttle-GFP-PSMA. The recombinant shuttle plasmid, pShuttle-GFP-PSMA and empty vector, pShuttle-CMV-EGFP were digested by SfiI and, by
PCR amplification, two fragments of $\sim 2.2 \mathrm{~kb}$ (consistent with the size of the inserted fragment) and $\sim 5.1 \mathrm{~kb}$ were obtained from the pShuttle-GFP-PSMA plasmid, while only the 5.1-kb fragment (recombinant shuttle vector fragment) was obtained from the empty vector (Fig. 1). The results of DNA sequencing were consistent with the reports obtained from GenBank: PSMA (NC_004463.1; http://www.ncbi.nlm.nih. gov/gene/1054986) and FOLH1 (NC_000011.10; http://www. ncbi.nlm.nih.gov/gene/2346).

Identification of recombinant adenovirus vector, pAdxsi-GFP-PSMA. The recombinant shuttle plasmid, 


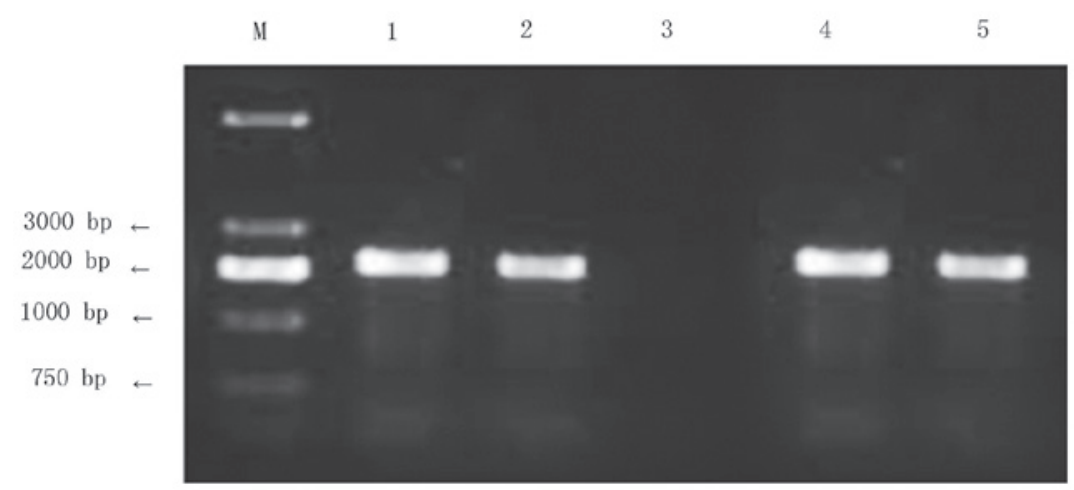

Figure 3. Polymerase chain reaction electrophoresis of purified recombinant adenovirus. Lane M, molecular weight marker, DL2000; lanes 1 and 2 , pShuttle-EGFP-PSMA/pAdxsi-GFP-PSMA plasmid (amplification results of PSMA-SfiI-F and PSMA-SfiI-R primers); lane 3, Ad-GFP virus (amplification results of PSMA-SfiI-F and PSMA-SfiI-R primers); lanes 4 and 5, Ad-GFP-PSMA adenovirus (amplification results of PSMA-SfiI-F and PSMA-SfiI-R primers). PSMA, prostate-specific membrane antigen; EGFP, enhanced green fluorescent protein.

pShuttle-GFP-PSMA and empty vector, pShuttle-CMV-EGFP were digested by SfiI, and loaded onto $1 \%$ agarose gel for electrophoresis. The results are presented in Fig. 2.

Construction and identification of the human recombinant adenovirus carrying the PSMA gene. pAdxsi-GFP-PSMA was linearized with PacI for transfection into HEK293 cells. After one week of culture, when the transfected cell showed obvious vacuole-like changes under a fluorescent microscope, the virus DNA was extracted and amplified by PCR. The emergence of a 2,200-bp target band during electrophoresis indicated successful recombination of the target genes into the adenovirus genome (Fig. 3). Subsequent to identification, the concentrated virus solution was used for re-infection of the HEK293 cells at various concentrations and the virus titer was $2.0 \times 10^{10} \mathrm{pfu} / \mathrm{ml}$.

Efficiency of in vitro transfection of the adenovirus into the $D C s$. Results of flow cytometry (Fig. 4) demonstrate that the transfection efficiency reached a maximum value $(89.84+1.13 \%)$ at an MOI value of 200, which was significantly different from that at MOI values of 100, 150, 300 and $400(\mathrm{P}<0.05)$. In addition, the apoptosis rate of the DCs was significantly lower in the MOI 200 group $(6.29+0.14 \%)$ when compared with the other groups $(\mathrm{P}<0.05)$, indicating a minimum impact on $\mathrm{DC}$ vitality at an MOI value of 200. Therefore, the optimal MOI value was determined to be 200 . When the MOI value was fixed at 200, the GFP expression level was observed under a fluorescent microscope 12, 24 and $48 \mathrm{~h}$ after transfection. The results demonstrated that GFP expression was greatest (up to $85 \%) 48 \mathrm{~h}$ following transfection (Fig. 5A-D).

Changes in immune function of DC after transfection with PSMA gene recombinant adenovirus

Morphology of DCs observed by fuorescence microscopy. The DCs were observed on different days under a fluorescence microscope. On day 1, cells were adherent, small, irregularly shaped (with small pseudopodia and no branch-like protrusions) and showed a uniform distribution of cell colonies. On day 5, the majority of cells became larger, semi-adherent irregular spindle cells that clustered and showed bacteria curtain-like protrusions on the periphery. On the seventh day after Ad-GFP transfection, the cell size increased, the spikes on the cell rim became more apparent with obvious branches, and the cells were star- or spindle-shaped, with obvious nuclei and grew in clusters. All of these features were similar to those of DCs, which had been cultured for seven days under normal conditions (Fig. 6A-D).

Expression levels of PSMA in DCs observed by western blotting. Following transfection ( $48 \mathrm{~h}$ ), the positive expression rate of GFP was $85 \%$. Western blot analysis revealed a band at around $110 \mathrm{kd}$, which is consistent with the size of PSMA proteins; however, no such band was observed in the control group (Fig. 7).

Expression of co-stimulatory molecules in DCs in each group following transfection. Flow cytometry revealed that immature DCs on day 5 expressed a reduced quantity of co-stimulatory and major histocompatibility complex class II (MHC II) molecules (Table I). On day 8, the DCs in the transfection and non-transfection groups all showed features of mature DCs (high expression of the co-stimulatory and MHC II molecules); the expression levels of CD80, CD83, CD86 and HLA-DR were significantly higher in the transfected DCs than in the non-transfected DCs $(\mathrm{P}<0.05)$. However, no difference was observed between the Ad-PSMA-DC and Ad-GFP-DC groups $(\mathrm{P}>0.05)$. The results demonstrate that transfection of the adenovirus promoted DC maturation and significantly upregulated the expression levels of co-stimulatory molecules.

Detection of IL-12 in the supernatant of each group. Following transfection ( $48 \mathrm{~h}$ ), the supernatant of each group was collected, and the quantity of IL-12 in the supernatant was measured by ELISA (Fig. 8). The IL-12 level in the Ad-PSMA-DC $(79.51 \pm 1.60 \mathrm{pg})$ and Ad-GFP-DC $(69.67 \pm 1.43 \mathrm{pg})$ groups was significantly higher than that in the non-transfection (normal-DC) group $(28.88 \pm 2.97 \mathrm{pg} ; \mathrm{P}<0.05)$; however, no significant difference was identified between the two transfected groups (Ad-PSMA-DC and Ad-GFP-DC). These results indicate that adenovirus transfection significantly promotes the ability of DCs to secrete IL-12, regardless of whether the cell carries the PSMA gene.

Cytotoxicity of effector cell (CTL) to target cells (LNCap, Dul45 and 22RV). A CCK-8 assay was conducted to detect the OD value of each well and the cytotoxicity of CTL in 
Table I. Phenotype analysis of DCs in each group on days 5 and 8.

\begin{tabular}{lcccc}
\hline & \multicolumn{4}{c}{ DC marker expression (\%) } \\
\cline { 2 - 5 } Group & CD80 & CD83 & CD86 & HLA-DR \\
\hline 5d-DCs & $22.95 \pm 3.34$ & $18.93 \pm 1.65$ & $29.64 \pm 0.83$ & $29.17 \pm 0.81$ \\
Ad-PSMA-DC & $33.29 \pm 1.13^{\mathrm{b}}$ & $36.02 \pm 0.71^{\mathrm{b}}$ & $53.30 \pm 0.86^{\mathrm{b}}$ & $53.00 \pm 1.62^{\mathrm{b}}$ \\
Ad-GFP-DC & $29.68 \pm 0.61^{\mathrm{c}}$ & $32.62 \pm 1.07^{\mathrm{c}}$ & $50.70 \pm 0.83^{\mathrm{c}}$ & $49.29 \pm 0.53^{\mathrm{c}}$ \\
8d-DCs & $28.27 \pm 1.04^{\mathrm{a}}$ & $28.08 \pm 1.16^{\mathrm{a}}$ & $41.05 \pm 1.33^{\mathrm{a}}$ & $46.87 \pm 1.12^{\mathrm{a}}$ \\
\hline
\end{tabular}

${ }^{a} \mathrm{P}<0.05$ vs. $5 \mathrm{~d}-\mathrm{DC}$ group; ${ }^{\mathrm{b}} \mathrm{P}<0.05,{ }^{\mathrm{c}} \mathrm{P}<0.01$ vs. 8d-DC group. DC, dendritic cell; $\mathrm{CD}$, cluster of differentiation; 5d-DC, immature DCs (day 5); PSMA, prostate-specific membrane antigen; GFP, green fluorescent protein; 8d-DC, mature DCs (day 8).
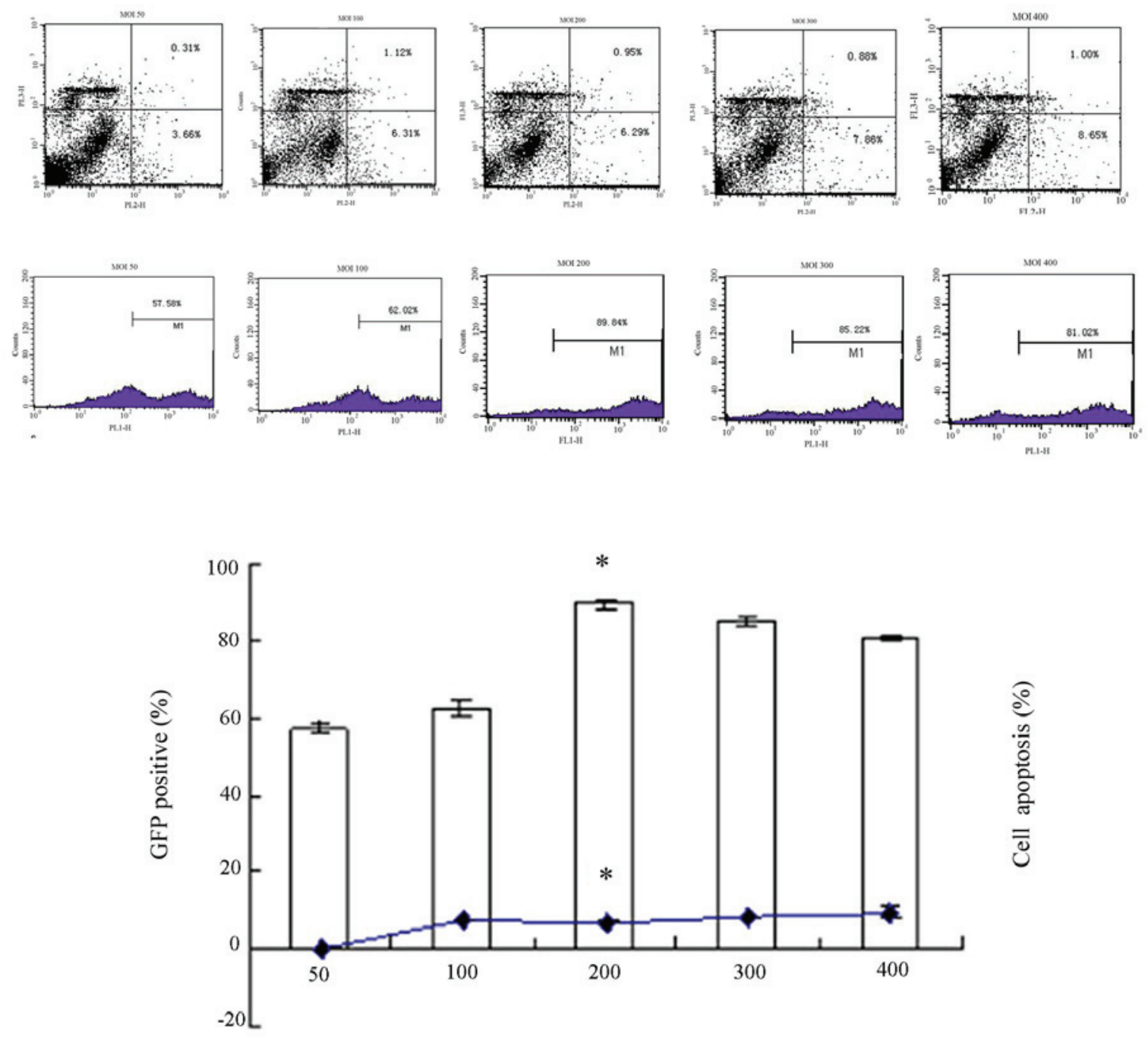

GFP positive (\%) $\rightarrow$ Cell apoptosis (\%)

Figure 4. Dendritic cell apoptosis rate at various MOI as evaluated by flow cytometry. As the MOI increased, the rate of apoptosis also increases. At the value of 200, the apoptotic rate of the cells was the highest. "P<0.05. MOI, multiplicity of infection; GFP, green fluorescent protein.

each group was calculated based on the following formula: Killing rate $(\%)=\left[1-\left(\mathrm{OD}_{\text {experimental group }}-\mathrm{OD}_{\text {effector cells alone }}\right) / \mathrm{OD}_{\text {target }}\right.$ cell alone $\mathrm{x} 100$. Statistical analysis revealed that CTLs that were stimulated by transfected DCs were associated with a stronger killing rate against all three $\mathrm{PCa}$ lines, when compared with CTLs that were stimulated by normal cultured DCs or CTLs 
A

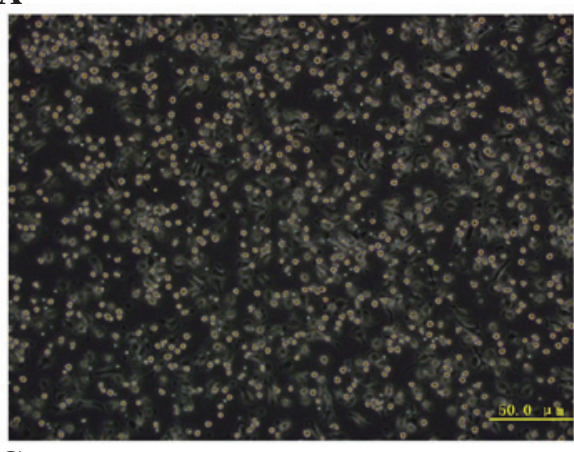

C

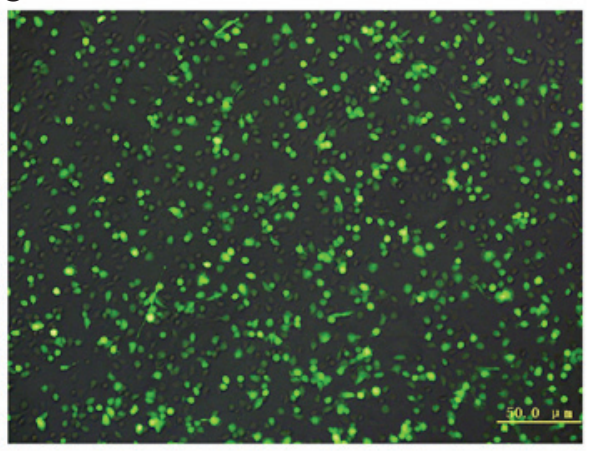

B

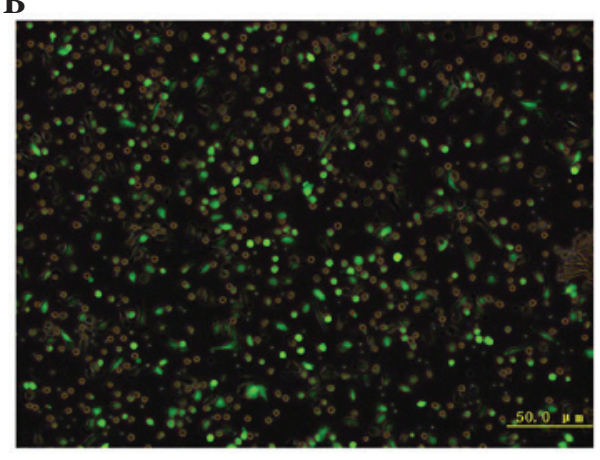

D

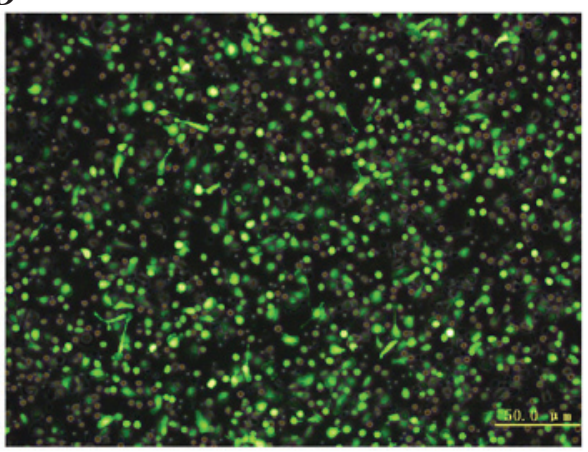

Figure 5. Transfection efficiency (reflected by green fluorescent protein expression) at a multiplicity of infection value of 200 at (A) 0 , (B) 12 , (C) 24 and (D) 48 h. (merged, x100).

A

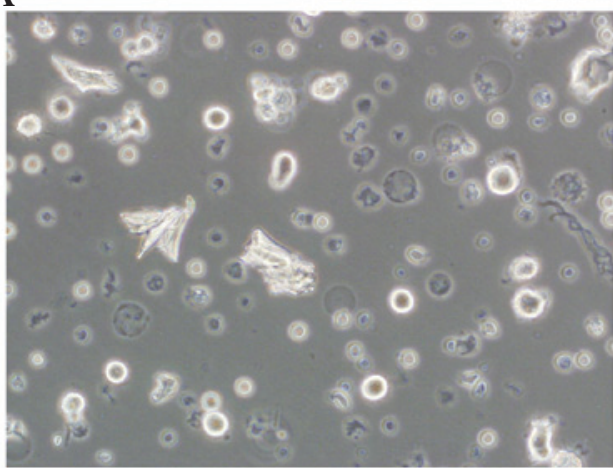

C

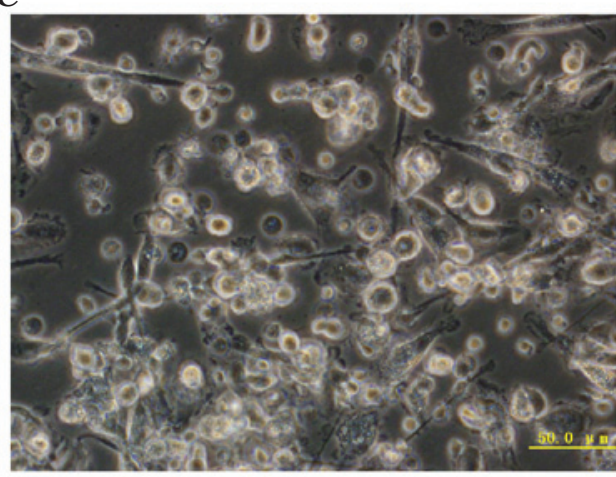

B

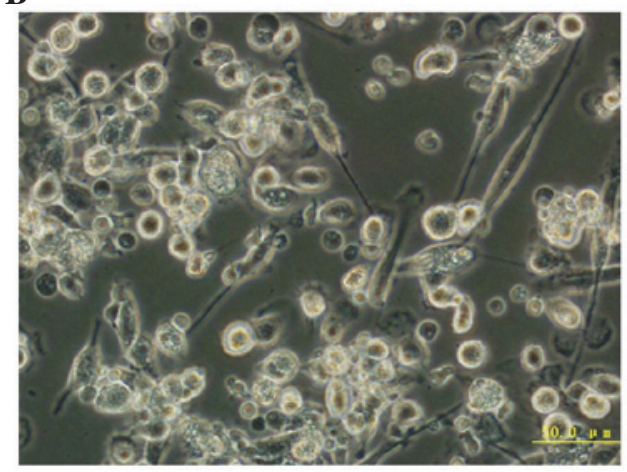

D

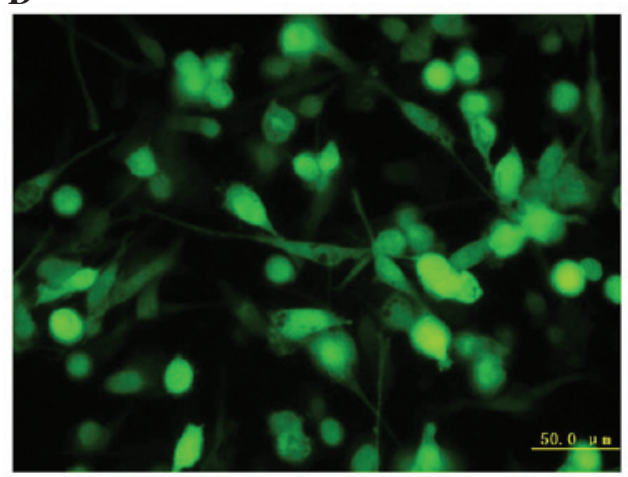

Figure 6. Morphology of normal cultured DCs on (A) day 1, (B) day 5 and (C) day 7, and (D) ad-GFP-transfected DCs on day 7 (magnification, x400). DC, dendritic cell; GFP, green fluorescent protein.

alone. Cytotoxicity reached a maximum value in all of the groups when the E:T ratio was 40:1. Furthermore, at the same E:T ratio, the killing rate of CTL in the Ad-PSMA-DC-CTL group against the LNCap cells (exhibiting high PSMA expression levels) was significantly higher than that against the Du145 and $22 R V$ cells $(P<0.05)$. In addition, the killing rate in the 

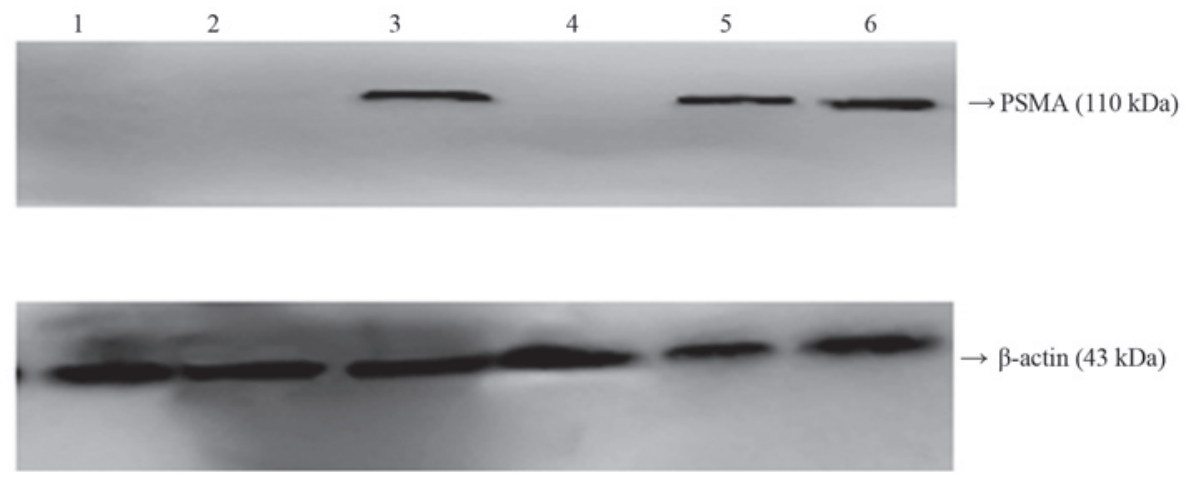

Figure 7. Detection of PSMA expression by western blot analysis. Lanes 1 and 2, DCs transfected with Ad-GFP virus; lane 3, positive control (LNCap cells); lane 4, negative control (HEK293 cells); lanes 5 and 6, DCs transfected with Ad-GFP-PSMA adenovirus. PSMA, prostate-specific membrane antigen; DC, dendritic cell; GFP, green fluorescent protein.

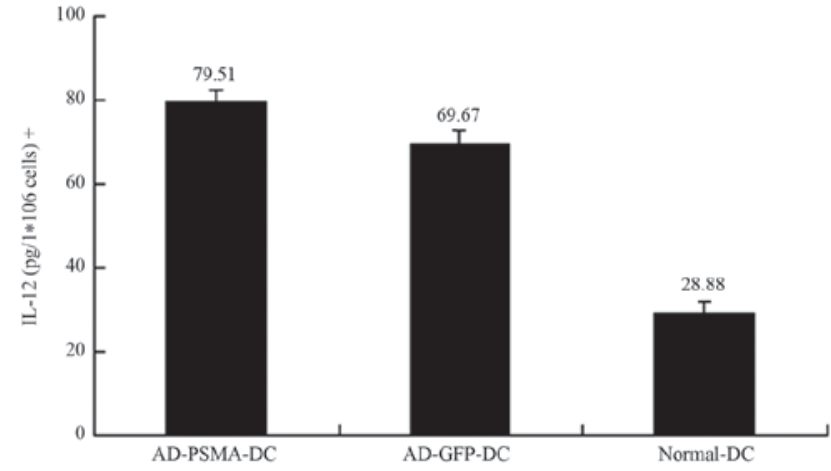

Figure 8. Detection of IL-12 level in the supernatant of each group by ELISA. The levels of IL-12 were higher in the AD-PSMA-DC group, compared with the other groups. Data are presented as the mean. IL, interleukin; PSMA, prostate-specific membrane antigen; DC, dendritic cell; GFP, green fluorescent protein.

Ad-PSMA-DC-CTL group was significantly higher than that in the Ad-GFP-DC-CTL, normal DC-CTL and CTL alone groups (Fig. 9A-C; $\mathrm{P}<0.05$ and Table II). The results demonstrate that DCs transfected with PSMA were more effective in eliciting the differentiation of tumor-specific CTLs, which exert a particularly potent killing effect on $\mathrm{PCa}$ cells with high PSMA expression levels, such as LNCap cells.

\section{Discussion}

PSMA is a type II transmembrane glycoprotein, which is secreted by prostate epithelial cells. It is composed of 750 amino acids, and has a relative molecular mass of $\sim 100,000$. There are three PSMA domains, comprising the intracellular $\mathrm{N}$-terminal, and the transmembrane and extracellular regions. On the intracellular and extracellular regions, there are a plurality of epitopes that may be combined with a variety of monoclonal antibodies (18). Compared with PSA and PAP, PSMA is regarded as a more sensitive and a more specific tumor maker. Its highly specific expression in PCa tissues has established PSMA as one of the leading targets for use within targeted therapies (19). Based on the above-mentioned information, a replication-defective adenoviral vector containing PSMA gene sequences was constructed for use in the present study.
The Adxsi system, which comprised a shuttle plasmid vector system, a virus backbone plasmid and the packaging cell line, HEK 293 was selected. The primary advantage of the Adxsi viral packaging system is that the packaged virus is stable and of a high titer, furthermore, the system may be used in the packaging of cytotoxic genes, as well as genes that influence adenovirus physiological processes, such as infection, duplication and packaging (20). The PSMA gene was amplified by PCR and the pShuttle-EGFP-PSMA was directly ligated into the viral plasmid according to a dual restriction site (I-Ceul and I-Scel) directional cloning method. There are few restriction sites of I-Ceul and I-Scel in the human genome, therefore, they can be used to identify longer gene sequences. Additionally, the application of HEK293 cells for the control of PSMA gene expression has improved the efficacy of virus packaging and the virus titer (21). $\mathrm{CsCl}$ density gradient centrifugation was performed for purification of the HEK293 cells, including exemption of defective viral particles, cellular debris and a small quantity of medium components, so as to maximize the reduction of pollution-induced immunological responses $(22,23)$ and to obtain a high titer $\left(2.0 \times 10^{10} \mathrm{pfu} / \mathrm{ml}\right)$ PSMA adenovirus with high productivity.

DCs are considered to be the most powerful antigen-presenting cells currently known. They are derived from hematopoietic stem cells, and are widely distributed in tissues and organs, but not in the brain. However, the number of DCs in the human body is limited ( $<1 \%$ in human PBMCs), with cancer patients possessing even fewer DCs, which are functionally deficient, as they are incapable of effective antigen presenting (24). In addition, the low immunogenicity of tumor cells and their lack of specific antigens has consistently presented a major issue during vaccine therapy in the activation of human immune responses (25).

There are three stages involved in the in vitro culture of DCs: i) Amplification, ii) differentiation induction and iii) maturation promotion. In the present study, PBMCs were enriched for the induction of DCs in the presence of IL-4, GM-CSF and TNF- $\alpha$. These cytokines inhibit the transformation of PBMCs into precursor cells, and also induce the generation and maturation of DCs $(26,27)$. However, due to the immune surveillance effect, and strong antigen uptaking and processing capacity of immature DCs, the 
Table II. Killing rate of effector cells (CTLs) against three prostate cancer cell lines.

Killing rate $(\%)$

Cell line

(E:T)

Ad-PSMA-DC-CTL

Ad-GFP-DC-CTL

Normal DC-CTL

CTL

LNCap

10:1

$72.18 \pm 0.43^{\mathrm{a}, \mathrm{b}}$

$64.28 \pm 1.63$

$63.96 \pm 1.47^{\mathrm{a}}$

$74.51 \pm 0.85^{\mathrm{a}}$

$37.90 \pm 1.39^{\mathrm{a}}$

20:1

$80.87 \pm 1.50^{\mathrm{a}, \mathrm{b}}$

$76.92 \pm 0.92^{\mathrm{a}}$

$78.75 \pm 0.99^{\mathrm{a}}$

$51.08 \pm 0.86^{\mathrm{a}}$

40:1

$89.76 \pm 0.44^{\mathrm{a}}$

$80.97 \pm 1.12$

$62.35 \pm 0.97^{\mathrm{a}}$

DU145

$\begin{array}{rl}10: 1 & 66.61 \pm 0.95^{\mathrm{a}} \\ 20: 1 & 74.49 \pm 1.10^{\mathrm{a}} \\ 40: 1 & 80.21 \pm 1.20^{\mathrm{a}} \\ 22 \mathrm{RV} & \\ 10: 1 & 68.16 \pm 0.82^{\mathrm{a}} \\ 20: 1 & 76.15 \pm 0.71^{\mathrm{a}} \\ 40: 1 & 83.50 \pm 0.85^{\mathrm{a}}\end{array}$

$63.22 \pm 1.86$

$60.83 \pm 1.03$

$35.81 \pm 0.76$

$66.96 \pm 0.82$

$64.73 \pm 1.68$

$40.90 \pm 1.10$

$76.66 \pm 0.37$

$73.52 \pm 1.29$

$51.84 \pm 2.24$

$58.81 \pm 0.48$

$56.34 \pm 1.23$

$29.85 \pm 0.38$

$71.52 \pm 0.74$

$33.46 \pm 1.38$

$79.66 \pm 0.64$

$71.87 \pm 1.75$

$37.98 \pm 0.49$

${ }^{\mathrm{a}} \mathrm{P}<0.05$, vs. Ad-GFP-DC-CTL; ${ }^{\mathrm{b}} \mathrm{P}<0.01$, vs. CTL. E, effector; T, target; CTL, cytotoxic T lymphocyte; PSMA, prostate-specific membrane antigen; DC, dendritic cell; GFP, green fluorescent protein. DC, dendritic cell; PSMA, prostate-specific membrane antigen; GFP, green fluorescent protein; CTL, cytotoxic T lymphocyte.

A

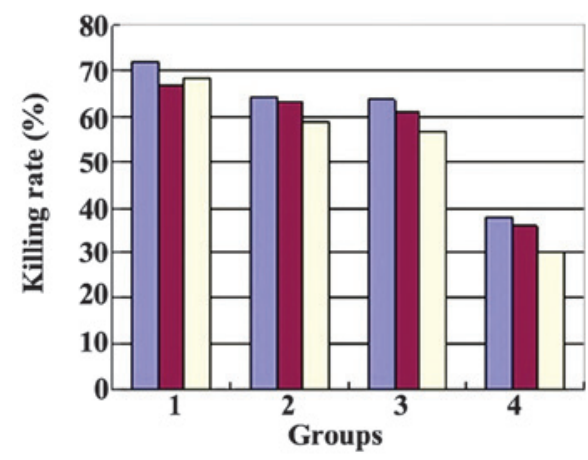

B

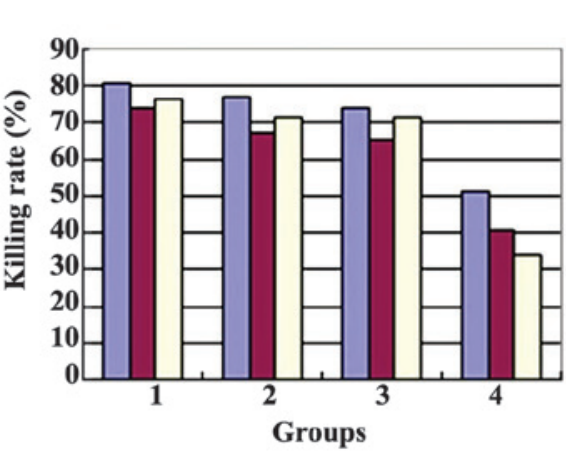

C

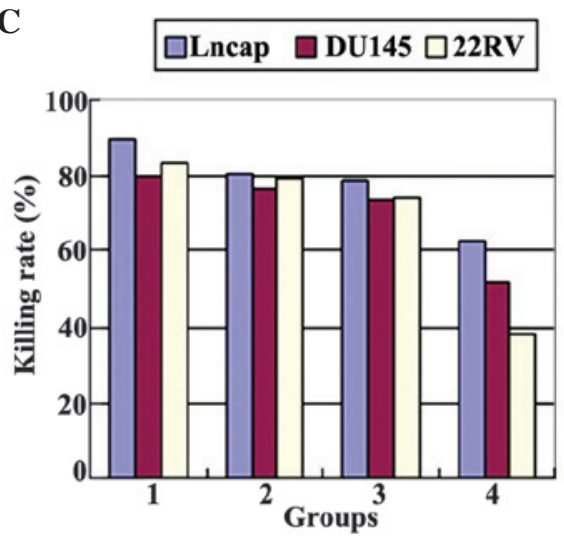

Figure 9.Cytotoxicity of DC vaccine in each group against cancer cells at different effector-to-target ratios: (A) 10:1; (B) 20:1; and (C) 40:1.1,AD-PSMA-DC-CTL; 2, AD-GFP-DC-CTL; 3, normal-DC-CTL; 4, CTL. DC, dendritic cell; PSMA, prostate-specific membrane antigen; GFP, green fluorescent protein; CTL, cytotoxic T lymphocyte.

adenovirus-carrying PSMA gene was added on the fifth day of culture to promote DC maturation and to obtain abundant specific tumor antigen-loaded DCs (28). The present study has demonstrated the validity of this method of constructing DC vaccines that are specific to PCa.

As a result of the high expression levels of MHC I and II molecules, co-stimulatory molecules (such as CD80 and CD86), adhesion molecules (such as CD44 and CD54, integrin $\beta 1$ and integrin $\beta 2$ ) and characteristic markers (such as CD83), the predominant role of mature DCs is the induction of immune activation. The mechanism of the strong immune stimulatory capacity of DCs is via an interaction between DCs and heat shock protein receptors (including CD91 and Toll-like receptors) on the DC surface, which triggers a series of reactions, including upregulation of surface factors, increased secretion levels of IL-12 and IFN- $\gamma$, promotion of DC maturation and improvement of antigen-presenting efficiency $(29,30)$.

In conclusion, in the current study, DCs induced by the PSMA-adenovirus exhibited significantly higher expression levels of CD80, CD86 and CD83, and a significantly higher secretion level of IL-12, when compared with that of DCs that had not been stimulated. However, no such difference was observed between the DCs induced by the PSMA-adenovirus or the empty virus. Furthermore, among all of the DC vaccines, the PSMA-DC vaccine demonstrated a more obvious cytotoxic effect against all three cancer cell lines (particularly against the LNCap cells) when compared with the other vaccines, including the adenovirus-DC vaccine. This indicated that the PSMA-targeted DC vaccine exerted a marked effect against the PCa cells; however, its ability to stimulate DC differentiation and maturation, and to improve their antigen-presenting 
efficiency was dependent on the loaded adenovirus. The above result may have been due to the expression of other co-stimulatory molecules that were not identified in the present study; therefore further studies are required to obtain a comprehensive perspective.

\section{Acknowledgements}

The present study was supported by a grant from the Liaoning Province Science and Technology Plan Projects (grant no. 2010225034).

\section{References}

1. Zumwalt $\mathrm{TH}$ and Goel A: Immunotherapy of metastatic colorectal cancer: prevailing Challenges and new perspectives. Curr Colorectal Cancer Rep 11: 125-140, 2015.

2. Zhang $\mathrm{H}$ and Huang B: Tumor cell-derived microparticles: a new form of cancer vaccine. Oncoimmunology 4: e1017704, 2015.

3. Onji M and Akbar SMF: On dendritic cell based therapy for cancers. Zhejiang Univ Sci B 6: 1-3, 2005.

4. Jemal A, Siegel R, Ward E, Hao Y, Xu J, Murray T and Thun MJ: Cancer statistics, 2008. CA Cancer J Clin 58: 71-96, 2008.

5. Gu F: Epidemiological survey of benign prostatic hyperplasia and prostate cancer in China. Chin Med J (Engl) 113: 299-302, 2000 (In Chinese).

6. Basler M and Groettrup M: Advances in prostate cancer immunotherapies. Drugs \& Aging 24: 197-221, 2007.

7. Murphy GP, Tjoa BA, Simmons SJ, Jarisch J, Bowes VA, Ragde H, Rogers M, Elgamal A, Kenny GM, Cobb OE, et al Infusion of dendritic cells pulsed with HLA-A2-specific prostate-specific membrane antigen peptides: A phase II prostate cancer vaccine trial involving patients with hormone-refractory metastatic disease. Prostate 38: 73-78, 1999.

8. Murphy GP, Tjoa BA, Simmons SJ, Rogers MK, Kenny GM and Jarisch J: Higher-dose and less frequent dendritic cellinfusions with PSMA peptides in hormone-refractory metastatic prostate cancer patients. Prostate 43: 59-62, 2000.

9. Zhou Y, Wang HP and Wang Q: Advances in design strategy of dendritic cell vaccine. Medical Journal of Chinese People's Liberation Army 31: 1205-1206, 2006 (In Chinese).

10. Ribas A, Butterfield LH, Glaspy JA and Economou JS: Cancer immunotherapy using gene-modified dendritic cells. Curr Gene Ther 2: 57-78, 2002.

11. Wu Q, Xia D, Carlsen S and Xiang J: Adenovirus-mediated transgene-engineered dendritic cell vaccine of cancer. Curr Gene Ther 5: 237-247, 2005.

12. Perner S, Hofer MD, Kim R, Shah RB, Li H, Möller P, Hautmann RE, Gschwend JE, Kuefer R and Rubin MA: Prostate specific membrane antigen expression as a predictor of prostate cancer progression. Hum Pathol 38: 696-701, 2007.

13. Zhang P, Chiu YC, Tostanoski LH and Jewell CM: Polyelectrolyte multilayers assembled entirely from immune signals on gold nanoparticle templates promote antigen-specific $\mathrm{T}$ cell response. ACS Nano 9: 6465-6477, 2015.

14. Qiu K, Yu B, Huang H, Zhang P, Ji L and Chao H: Tetranuclear ruthenium(II) complexes with oligo-oxyethylene linkers as oneand two-photon luminescent tracking non-viral gene vectors. Dalton Trans 44: 7058-7065, 2015.
15. XiaD, MoyanaT and Xiang J: Combinational adenovirus-mediated gene therapy and dendritic cell vaccine in combating well-established tumors. Cell Res 16: 241-259, 2006.

16. Zhang Yan and Shen Xi: The impact of transfection with common viral vector on dendritic cells. Foreign Medical Science (Molecular Biology Section) 25: 188-190, 2003 (In Chinese).

17. Liu Qingxin and Song Jun: Viral vector-mediated gene therapy for prostate cancer. International Journal of Urology and Nephrology 20: 62-63, 2000 (In Chinese).

18. Slovin SF, Kehoe M, Durso R, Fernandez C, Olson W, Gao JP, Israel R, Scher HI and Morris S: A phase I dose escalation trial of vaccine replicon particles (VRP) expressing prostate-specific membrane antigen (PSMA) in subjects with prostate cancer. Vaccine 31: 943-949, 2013.

19. Wang W and Mo ZN: Advances in prostate-specific membrane antigen targeted therapies for prostate cancer. Zhonghua $\mathrm{Nan} \mathrm{Ke}$ Xue 16: 547-551, 2010 (In Chinese).

20. Medin JA, Liang SB, Hou JW, Kelley LS, Peace DJ and Fowler DH: Efficient transfer of PSA and PSMA cDNAs into DCs generates antibody and $\mathrm{T}$ cell antitumor responses in vivo. Cancer Gene Ther 12: 540-551, 2005.

21. Zeng H, Wu Q, Li H, Wei Q, Lu Y, Li X, Wang F, Zhao F, Ding Z and Yang Y: Construction of prostate-specific expressed recombinant plasmids with high transcriptional activity of prostate-specific membrane antigen (PSMA) promoter/enhancer. J Androl 26: 215-221, 2005.

22. Liu Q, Zaiss AK, Colarusso P, Patel K, Haljan G, Wickham TJ and Muruve DA: The role of capsid-endothelial interactions in the innate immune response to adenovirus vectors. Hum Gene Ther 14: 627-643, 2003.

23. Philpott NJ, Nociari M, Elkon KB and Falck-Pedersen E: Adenovirus-induced maturation of dendritic cells through a PI3 kinase-mediated TNF-alpha induction pathway. Proc Nat Acad Sci USA 101: 6200-6205, 2004.

24. Smyth MJ, Godfrey DI and Trapani JA: A fresh look at tumor immunosurveillance and immunotherapy. Nat Immunol 2: 293-299, 2001.

25. Fan Y and Moon JJ: Nanoparticle drug delivery systems designed to improve cancer vaccines and immunotherapy. Vaccines 3: 662-685, 2015.

26. Liu A, Takahashi M, Narita M, Zheng Z, Kanazawa N, Abe T, Nikkuni K, Furukawa T, Toba K, Fuse I and Aizawa Y: Generation of functional and mature dendritic cells from cord blood and bone marrow CD34+ cells by two-step culture combined with calcium ionophore treatment. J Immunol Methods 261: 49-63, 2002.

27. Hill JA, Ichim TE, Kusznieruk KP, Li M, Huang X, Yan X, Zhong R, Cairns E, Bell DA and Min WP: Immune modulation by silencing IL-12 production in dendritic cells using small interfering RNA. J Immunol 171: 691-696, 2003.

28. Weigel BJ, Panoskaltsis-Mortari A, Diers M, Garcia M, Lees C, Krieg AM, Chen W and Blazar BR: Dendritic cells Pulsed or fused with AML cellular antigen provide comparable in vivo antitumor protective responses. Exp Hematol 34: 1403-1412, 2006.

29. Rivoltini L, Castelli C, Carrabba M, Mazzaferro V, Pilla L, Huber V, Coppa J, Gallino G, Scheibenbogen C, Squarcina P, et al: Human tumor-derived heat shock protein 96 mediates in vitro activation and in vivo expansion of melanoma- and colon carcinoma-specific T cells. J Immunol 171: 3467-3474, 2003.

30. Mazzaferro V, Coppa J, Carrabba MG, Rivoltini L, Schiavo M, Regalia E, Mariani L, Camerini T, Marchianò A, Andreola S, et al: Vaccination with autologous tumor-derived heat-shock protein gp96 after liver resection for metastatic colorectal cancer. Clin Cancer Res 9: 3235-3245, 2003. 